\title{
A Mesoscopic Simulation Study on the Solubilization of Menthol by Platycodin D
}

\author{
Haiou Ding ${ }^{\mathrm{a}, 1}$, Xinyuan Shi ${ }^{\mathrm{b}, 1, *}$, Xingxing Dai ${ }^{\mathrm{b}}$, Qianqian Yin ${ }^{\mathrm{a}}$ and Yanjiang Qiao, \\ ${ }^{a}$ School of Traditional Chinese Medicine, Capital Medical University, Beijing, China, 100069 \\ ${ }^{b}$ Beijing University of Chinese Medicine, China, 100102 \\ ${ }^{I}$ These authors contributed equally to this work.
}

Received 15 May 2013; Accepted 25 July 2013

\begin{abstract}
Mesodyn simulation is employed in this research to investigate the influences of temperature on the solubilization of menthol by platycodin D. The results show that the crucial factor on the speed of solubilization of menthol is the time for micellization of platycodin D. Increase in temperature is not conducive to the micellization of platycodin D, but it may have a positive effect on the diffusion of menthol into platycodin D micelles. Considering both the speed and efficiency of the solubilization of menthol by platycodin $\mathrm{D}$, there exists an optimum temperature for the preparation process. This work offers abundant information at the mesoscopic level and may serve as guidance for the solubilization of volatile oil in medicine preparation.
\end{abstract}

Keywords: Mesodyn, menthol, platycodin D, temperature, solubilization

\section{Introduction}

Many Chinese herbs are rich in volatile oil component, which is insoluble in water and has a strong volatility. This will not only lead to the low bioavailability of these components and stability problems of preparations but also restrict the development of new formulations.[1] Therefore, it is of great significance to explore how to improve and enhance the stability of volatile oil and bioavailability with the purpose of guaranteeing and improving the quality of volatile oil preparations. Saponins, a kind of plant based biosurfactant, which possess both surfactant properties and biological activities,[2-4] can increase the solubility of some hydrophobic components. The solubilization performance is associated with the physicochemical properties of saponin and solubilizer.[2,5-7]

Yinqiao Powder, a Traditional Chinese Medicine (TCM) prescription composed of Platycodon grandiflorum, Mentha haplocalyx and so on, is widely used for treating acute tonsillitis, faucitis, pneumonia and epidemic hemorrhagic fever.[8] The main active ingredients of them are platycodin and mint oil respectively, especially platycodin D (PD) and menthol (M).[9-11] It's reported that platycodin can increase the solubility of mint oil efficiently because of its micellization in aqueous solutions during the preparation process.[12] However, the micelle formation usually accomplishes in milliseconds. It is difficult to study solubilization process by common experimental methods. Furthermore, the solubilization of mint oil is strongly influenced by temperature,[13] but the related research is few and within a narrow temperature range. This is mainly

*E-mail address: 15810764862@163.com

ISSN: 1791-2377 @ 2013 Kavala Institute of Technology. All rights reserved. because that this kind of research is time-consuming and hard to operate with extreme experiment conditions.

Mesoscopic simulation has emerged as a powerful tool in the study of microstructures. It not only complements experimental work effectively with wide parameters, but also offers guidance for future experimental research. MesoDyn has been widely used in the investigation of the phase behaviors, such as phase separation and micelle growth kinetics.[14,15] And it also performs well in exploring the influence of temperature on changes of micelle morphology.[16] Thus, it may provide a deeper understanding for the solubilization of mint oil by platycodin.

In this work, we firstly determined the critical aggregation concentration (CAC) of $\mathrm{M}$ and the critical micelle concentration (CMC) of PD in aqueous solution, and then we investigated the solubilization process of $\mathrm{M}$ by PD. The effects of temperature that closely related to the process of preparation were also studied by MesoDyn simulation to provide mesoscale information for further study on the solubilization mechanism and to offer guidance to improve the efficiency of experiments.

\section{Method, model, parameters and simulation conditions}

The basic idea of MesoDyn simulation is the density functional theory. It states that the Helmholtz free energy $\mathbf{F}$ of an inhomogeneous liquid is a function of the local density function $\boldsymbol{\rho}$. All thermodynamic functions can be derived from it.[17-20] The model used in the MesoDyn method consists of beads of various types. Each bead is a specific component type representing covalently bonded groups of atoms. The interactions between beads can be described by harmonic oscillator potentials for the intramolecular 
interactions (Gaussian chain) and a mean field potential for all other interactions.[21]

The components being used in this simulation comprise of PD, M and water. The coarse-grained models are shown in Fig.1. PD is divided into three types of particles (A, B, and $\mathrm{G}$ ) according to the polarity of the group they represent. $M$ is represented by bead $M$ and water is represented by bead W.

The MesoDyn input parameter $\lambda_{i j}$ is related to the effect Flory-Huggins parameters via $\lambda_{i j}=\chi_{i j} R T$, where $\chi_{i j}$ can be derived from the solubility parameters $\boldsymbol{\delta}$, which can be calculated by Amorphous Cell and Discover modules in Materials Studio 4.1 (Accelrys Inc.). The interaction parameters used in the simulation are given in Table 1.

Considering the simulation quality and computation efficiency, the simulations were performed in a $32 \times 32 \times 32$ cubic box with periodic boundary condition. Time step is $10.0 \mathrm{~ns}$, number of steps is $100,000(1.0 \mathrm{~ms})$. The grid spacing of $1.0 \mathrm{~nm}$ was taken for the simulation. To ensure isotropy of all grid-restricted operators, the bond length is set to be $1.1543 \mathrm{~nm}$. [18,22] Bead diffusion coefficient is set to be $1.0 \times 10^{-7} \mathrm{~cm}^{2} \cdot \mathrm{s}^{-1}$ in order to ensure a stable numerical algorithm. Noise parameter is taken to be 75.002 .

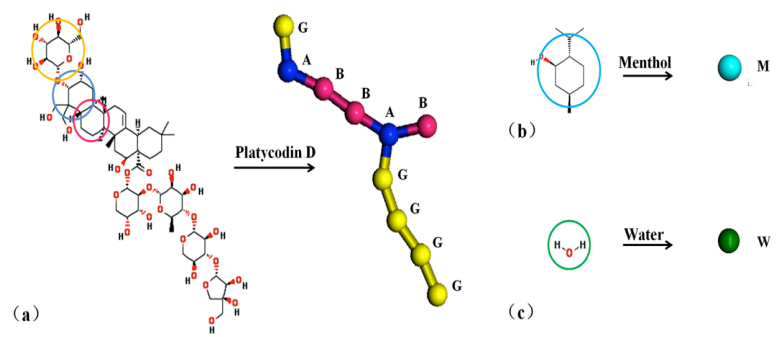

Fig. 1. Coarse-grained models. (a) PD; (b) M; (c) water.

Table.1 Interaction parameters $\lambda$ ij used in MesoDyn simulations (unit: $\mathrm{KJ} / \mathrm{mol}$ )

\begin{tabular}{llllll}
\hline & W & G & A & B & M \\
\hline W & 0 & & & & \\
G & 12.1 & 0 & & & \\
A & 33.4 & 5.6 & 0 & & \\
B & 52.4 & 23.3 & 7.7 & 0 & \\
M & 69.4 & 26.1 & 7.8 & 0.1 & 0 \\
\hline
\end{tabular}

\section{Results and Discussion}

\subsection{Determination on CAC of $M$ and $C M C$ of $P D$ in aqueous solution}

In Mesodyn simulation, the isodensity surface and density profile reveal the structure features and order parameter captures the effects of phase separation. Order parameters with large values indicate strong phase segregation. [23,24]. We set temperature at $298 \mathrm{~K}$ in this section. As showed in Fig.2, the CAC of $\mathrm{M}$ was $1.0 \mathrm{vol} \%$, $\mathrm{M}$ began to segregate from water and form into a spherical aggregation at this concentration. It indicates that $\mathrm{M}$ is hard-to-dissolve and need to be encapsulated by solubilizer in aqueous solution. As biosurfactants, PD will form into micelles over critical micelle concentration (CMC) in aqueous solutions and the micelles can be used to load hydrophobic components. For brevity and clarity, we chose the order parameter of bead G to represent the phase segregation of PD in this simulation study. According to the simulation result, the CMC of PD was $0.3 \mathrm{vol} \%$ as showed in Fig.3. It states that PD can function as solubilizer at low concentration. It is profitable for the preparation in terms of the economic aspect

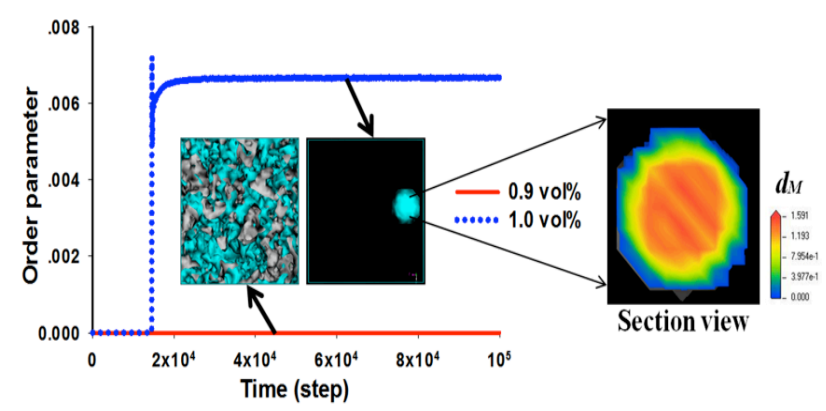

Fig. 2 Determination on the CAC of $\mathrm{M}$ in aqueous solution. Red line: order parameter of $\mathrm{M}$ in $0.9 \mathrm{vol} \% \mathrm{M} /$ water system; blue dot line: order parameter of $\mathrm{M}$ in $1.0 \mathrm{vol} \% \mathrm{M} /$ water system. Pictures presented here are isodensity surfaces of $M$ at equilibrium state for the two systems respectively. Section view is the density profile of $\mathrm{M}$ in $\mathrm{M}$ aggregate.

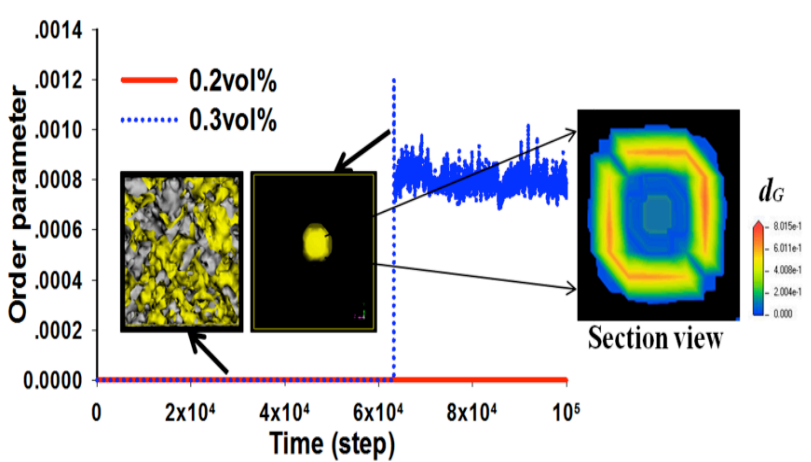

Fig.3 Determination on the CMC of PD in aqueous solution. Red line; order parameter of $\mathrm{G}$ in $0.2 \mathrm{vol} \% \mathrm{PD} /$ water system; blue dot line: order parameter of $\mathrm{G}$ in $0.3 \mathrm{vol} \% \mathrm{PD} /$ water system. Pictures presented here are isodensity surfaces of $\mathrm{G}$ at equilibrium state for the two systems respectively. Section view is the density profile of $G$ in PD micelle, which illustrates that the beads G of PD form into the hydrophilic shell of the micelle

\subsection{Dynamic solubilization process of $M$ by $P D$}

The dynamic solubilization process of $\mathrm{M}$ by $\mathrm{PD}$ was observed in $1 \mathrm{vol} \% / 3 \mathrm{vol} \% \mathrm{M} / \mathrm{PD} /$ water system at $298 \mathrm{~K}$. (Fig.4). The order parameters indicated that the solubilization process could be divided into four stages (Fig.4a) and the corresponding morphologies (Fig.4b) were showed below. During the first stage (0 100steps), the system was in a homogeneous state. In the second stage (101 $\sim 164$ steps), the order parameters of $\mathrm{G}$ took on a rapid increase which stated the micellization of PD; while the order parameters of $\mathrm{M}$ varied little which demonstrated that M still dissolved evenly in water. The stage three (165 595steps) contained two parts. In part one (165 435steps), the order parameters of $G$ decreased, the rough micelles formed in the previous stage were repaired; the order parameters of $M$ began to increase, $M$ segregated from water, part of $\mathrm{M}$ diffused into the PD micelles and the rest formed into aggregations. In part two (436 595steps), the order parameters of $\mathrm{G}$ changed little indicated that $\mathrm{PD}$ micelles reached their equilibrium stage; the order parameters of $\mathrm{M}$ increased till reached its maximum, $\mathrm{M}$ were gradually diffused into PD micelles. In the fourth stage (595 100,000steps; to express the solubilization process clearly, order parameter of only first 1000 steps was showed 
in Fig.4a), the order parameters were largely maintained and the system was in a stable state. The site of menthol in PD micelle was showed in Fig.4c.

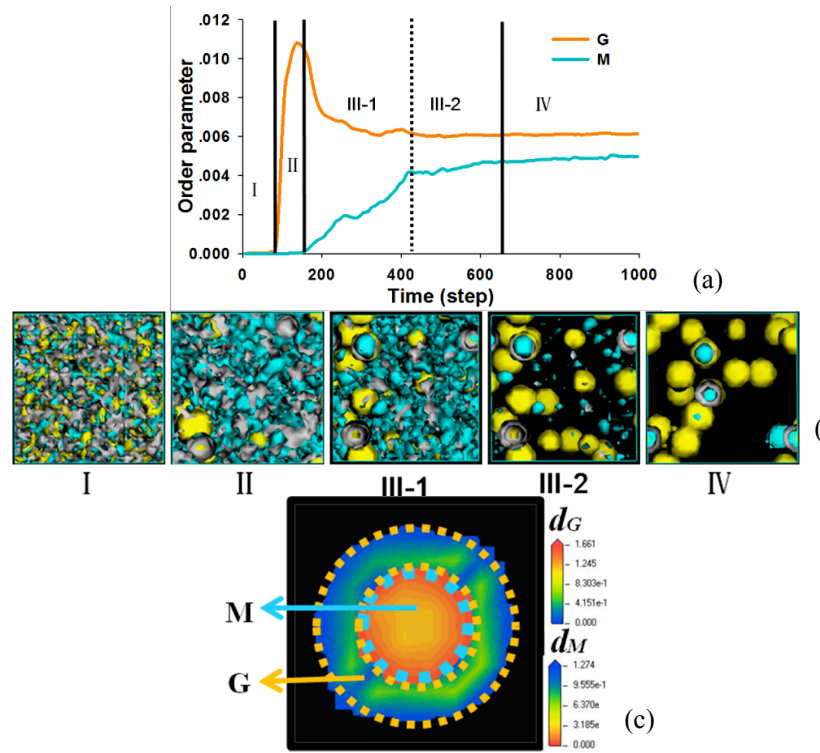

Fig.4 Dynamic solubilization process of $M$ by PD. (a) order parameters (b) isodensity surfaces, yellow: isodensity surface of G; blue: isodensity surface of M. (c) Section view is the density profile of $G$ and $M$ in $M$ loaded PD micelle, which figures out that $M$ is wrapped by the hydrophilic shell of PD micelle.

\subsection{Effect of temperature on the solubilization of $M$ by PD}

Temperature is an important factor during the preparation process. Taken the $1.0 \mathrm{vol} \% \mathrm{M} /$ water system, $3.0 \mathrm{vol} \%$ $\mathrm{PD} /$ water system and $1 \mathrm{vol} \% / 3 \mathrm{vol} \% \mathrm{M} / \mathrm{PD} /$ water system for instance, a comparison study on the solubilization of $\mathrm{M}$ by PD was carried out at the temperature ranging from $298 \mathrm{~K}$ to $373 \mathrm{~K}$. The time needed for aggregation, the order parameter of equilibrium state and morphologies were investigated. Given the structures have been explained in detail above, we only showed the isodensity surfaces to represent the morphologies here.

\subsubsection{Time for aggregation of $M$, micellization of $P D$ and} solubilization of $M$ by PD

Rising temperature has two opposite effects on the aggregation behavior of $1.0 \mathrm{vol} \% \mathrm{M} /$ water system. One is the weakening of hydration between hydroxyl of $M$ and water, it's the positive effect for $M$ to aggregate; the other is the promotion of thermal motion, it's the negative one [20]. From $298 \mathrm{~K}$ to $343 \mathrm{k}$, time for aggregation of $\mathrm{M}$ showed a decrease, this may because that the former was much pronounced during this temperature range. When temperature increased to $358 \mathrm{~K}$ and $373 \mathrm{~K}$, time for aggregation of $\mathrm{M}$ took on a sharp turn, this may attribute to the dominating effect of the latter.

Time for micellization of PD increased with temperature raising and obviously at high temperature $(358 \mathrm{~K}, 373 \mathrm{~K})$ in $3.0 \mathrm{vol} \% \mathrm{PD} /$ water system. That might because that the micelle formation was an exothermic hydration process. The increased temperature enhanced the thermal motion of PD molecules which made the system more difficult to reach equilibrium.

In 1vol $\% / 3$ vol $\% \mathrm{M} / \mathrm{PD} /$ water system, with temperature raised, time for both $\mathrm{PD}$ and $\mathrm{M}$ to aggregate shared the stepped increasing mode. Time for $M$ to aggregate was longer than PD's in the whole temperature range. It demonstrated that the solubilization of $\mathrm{M}$ by PD followed the procedure illustrated in section 3.2.

Through comparing the results above, we get following conclusions: high temperature is negative for the micellization of PD in terms of the speed; time for micellization of PD is the deciding factor on the speed of solubilization of $\mathrm{M}$ and $\mathrm{M}$ also has influence on the formation speed of PD micelles. Thus, in $1 \mathrm{vol} \% / 3 \mathrm{vol} \%$ $\mathrm{M} / \mathrm{PD} /$ water system, high temperature above $328 \mathrm{~K}$ is negative for micellization, which indicates that the temperature of solubilization process of M by PD shouldn't be above $328 \mathrm{~K}$. Simulation results for this section were showed in Fig. 5.

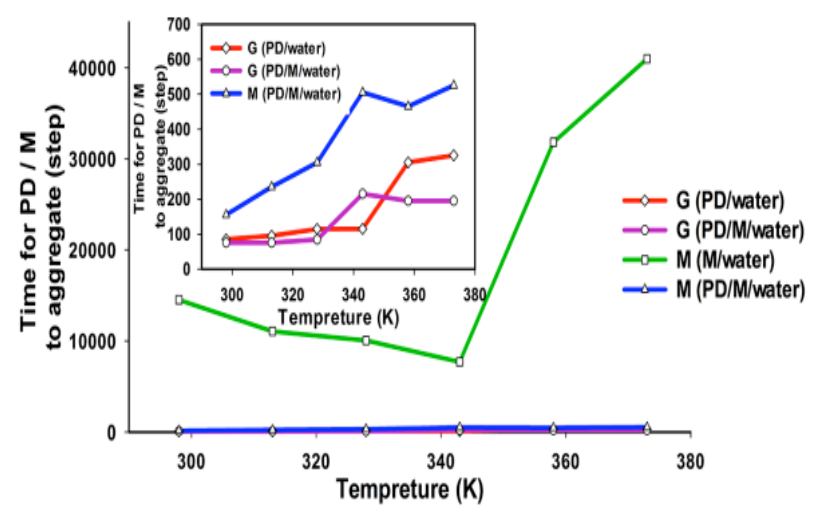

Fig.5 Evolution of time for aggregation of $\mathrm{M}$ and micellization of $\mathrm{PD}$ with temperature increasing.

\subsubsection{Order parameter and morphology of $M$ aggregates and PD micelles}

In $1.0 \mathrm{vol} \% \mathrm{M} /$ water system, order parameter of $\mathrm{M}$ decreased a little from $298 \mathrm{~K}$ to $373 \mathrm{~K}$ (Fig.6), the morphology changed little from $298 \mathrm{~K}$ to $358 \mathrm{~K}$, at $373 \mathrm{~K}$, with the water boiled, the aggregate of $\mathrm{M}$ began to split (Fig.7).

In 3.0vol\% PD/water system, the order parameter of $\mathrm{G}$ decreased gradually (Fig.6) and there was an increase in number and decrease in volume of PD micelles from 298K to $373 \mathrm{~K}$ (Fig.8). It states that the weakening in phase separation and indicates that rise in temperature is not beneficial for the formation of PD micelle.

In $1 \mathrm{vol} \% / 3 \mathrm{vol} \% \mathrm{M} / \mathrm{PD} /$ water system, order parameter of $\mathrm{M}$ varied little from $298 \mathrm{~K}$ to $313 \mathrm{~K}$, part of $\mathrm{M}$ diffused into PD micelles, the rest aggregated in water solution. At $328 \mathrm{~K}$, the order parameter decreased sharply and all the M diffused into PD micelles. With further growth of temperature, the order parameter changed a little and all the $\mathrm{M}$ were still encapsulated in PD micelles. The order parameter of $\mathrm{G}$ in $1 \mathrm{vol} \% / 3 \mathrm{vol} \% \mathrm{M} / \mathrm{PD} /$ water system got its maximum at $328 \mathrm{~K}$ and the PD micelle was big and least in number which accounted for the strongest phase separation at this temperature. (Fig.6, Fig.9).

Synthetically considering the simulation results, we may conclude that increase in temperature is not conducive to the aggregation of $\mathrm{M}$ and the micellization of $\mathrm{PD}$, but it may have a positive effect on the diffusion of $M$ into $P D$ micelles. Taking both the speed and efficiency of the solubilization of $\mathrm{M}$ into consideration, $328 \mathrm{~K}$ is the optimum temperature in the preparation process for this system. And at the highest temperature (373K) in this study, PD micelles still have high solubilizing capacity of $\mathrm{M}$. 


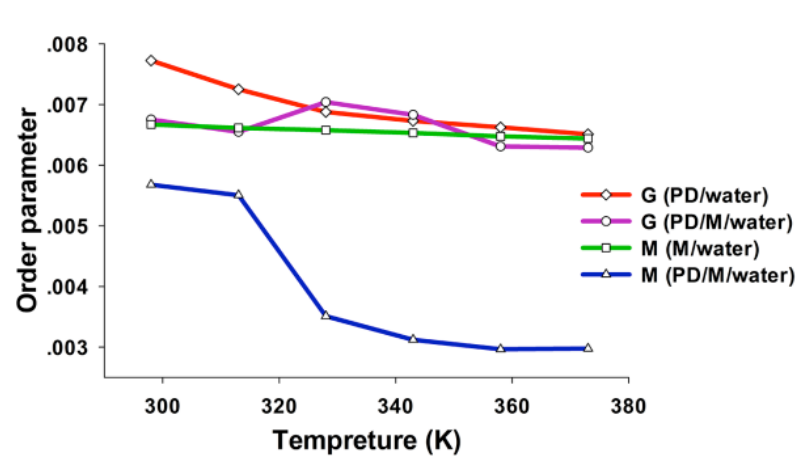

Fig.6 Evolution of order parameter of $\mathrm{G}$ or $\mathrm{M}$ with temperature increasing.

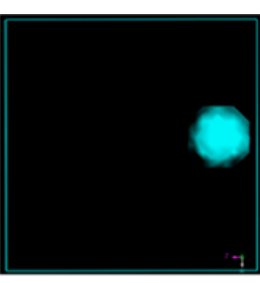

298K

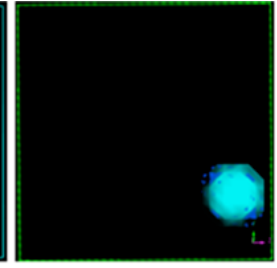

$358 \mathrm{~K}$

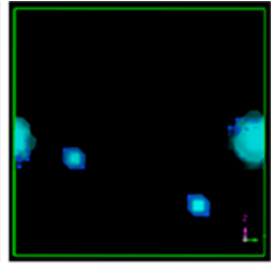

$373 K$
Fig.7 Morphology of M aggregate.

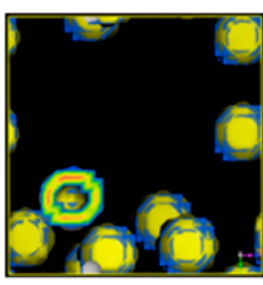

298K

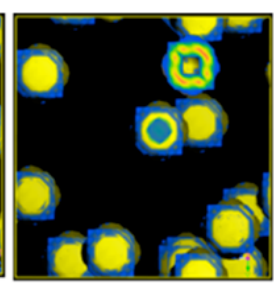

$328 \mathrm{~K}$

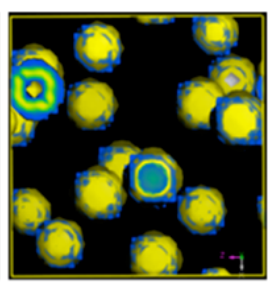

$373 K$

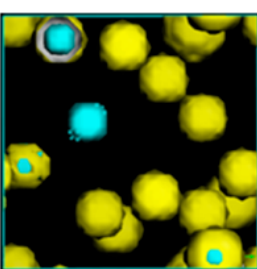

298K

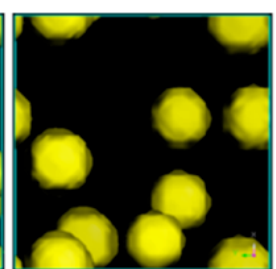

$328 \mathrm{~K}$

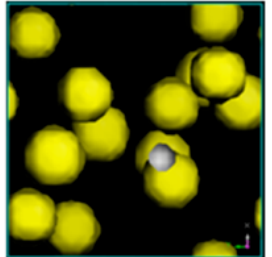

$373 K$
Fig.9 Morphology of M-loaded PD micelle.

\section{Conclusions}

Mesodyn method was adopted in this study, according to the simulation results, we got the following conclusions. Firstly, PD could function as solubilizer at low concentration and the solubilization of $\mathrm{M}$ by $\mathrm{PD}$ went through a "PD micelles formed and $\mathrm{M}$ diffused into" process. The determination factor on the speed of solubilization of $M$ was the time for PD to aggregate. Secondly, increase in temperature was not conducive to the micellization of $\mathrm{PD}$, but it may have a positive effect on the diffusion of $\mathrm{M}$ into $\mathrm{PD}$ micelles. Considering both the speed and efficiency of the solubilization of $\mathrm{M}$ in the preparation process, $328 \mathrm{~K}$ was the optimum temperature for this system. This simulation study supplemented the solubilizing mechanism at a mesoscopic level and achieved to explore the effect of temperature on the solubilization of menthol with wide temperature range. It may serve as guidance for the solubilization of volatile oil in medicine preparation.

\section{Acknowledgements}

This work was financially Supported by the National Natural Science Foundation of China (81073058) and Innovation Team Foundation of Beijing University of Chinese Medicine, Beijing (2011-CXTD-11, Research Center of TCM-information Engineering)

\section{References}

1. Wu G.T., Zhang X.H., Li F.Q., "Advances in pharmaceutical studies on improvement of stability of volatile oils of Chinese material medica", Pharm Care \& Res 8 (3), 2008, pp. 197-200.

2. Güçlü-Üstündağ Ö., Mazza G., "Saponins: properties, applications and processing”, Crit Rev Food Sci Nutr 47, 2007, pp. 231-258.

3. Sasaki Y., Mizutani K., Kasai R., et al, "Solubilizing Properties of Glycyrrhizin and Its Derivatives: Solubilization of Sakosaponin-a, the Saponi of Bupleuri Radix”, Chem Pharm Bull 36 (5), 1988, pp. 3491-3495.

4. Dai X.X., Shi X.Y., Wang Y.G., et al, "Solubilization of saikosaponin a by ginsenoside Ro biosurfactant in aqueous solution: Mesoscopic simulation”, J Colloid Interface Sci 384, 2012, pp. 73-80.

5. Schöpke T., Bartlakowski J., "Effects of saponins on the water solubility of quercetihn [quercetin]", Pharmazie. 52, 1997, pp. 232234.

6. Walthelm U., Dittrich K., Gelbrich G., et al, "Effects of saponins on the water solubility of different model compounds", Planta Med 67 (1), 2001, pp. 49-54.

7. Oakenfull D., "Aggregation of saponins and bile acids in aqueous solution", Aust J Chem 39, 1986, pp. 1671-1683.

8. Chen Q.M., Huang L.J., Wang W., "Clinical Application and Pharmacological Experiment Study of Yingqiao Powder", Hunan Guiding Journal of TCM 9 (9), 2003, pp. 37-39.

9. Zhao H.P., Liao Z.G., Zhu G.H., et al, "Discussion on the holistic reevaluation of the commercial Yinqiao San serial traditional Chinese patent medicines”, CJTCMP 27 (11), 2011, pp. 2779-2781.
10. Guo L., Zhang C., LI L., et al, “Advances in Studies on Platycodon grandiflorum”, China Journal of Chinese Materia Medica 32 (3), 2007, pp. 181-185.

11. Liang C.Y., Li W.L., Zhang H.Q., et al, "The Advance on the Research of Chemical Constituents and Pharmacological Activities of Mentha haplocalyx", Chinese Wild Plant Resources 22 (3), 2003, pp. 9-12.

12. Lin Y.L., Zhang J.M., 'Discussion on the research thought and method of the "meridian guiding" theory of Platycodon Root", Pharmacy and Clinics of Chinese Materia Medica 2 (2), 2011, pp. 50-52.

13. Chen Y.X., Ma J.G., Xu S.Y., "The System of Mint Oil Solubilized by Micelles", Journal of Wuxi University of Light Industry 20 (3), 2001, pp. 238-242.

14. Maurits N.M., Sevink G.J.A., Zvelindovsky A.V., et al, "Pathway controlled morphology formation in polymer systems: reactions, shear, and microphase separation”, Macromolecules 32, 1999, pp. 7674-7681.

15. Li Y.M., Xu G.Y., Wu D., et al, "The aggregation behavior between anionic carboxymethylchitosan and cetyltrimethylammonium bromide: MesoDyn simulation and experiments", Eur Polym J 43, 2007, pp. 2690-2698.

16. Lam Y.M., Goldbeck-Wood G., "Mesoscale simulation of block copolymers in aqueous solution: parameterisation, micelle growth kinetics and the effect of temperature and concentration Morphology", Polymer 44, 2003, pp. 3593-605. 
17. Fraaije J.G.E.M., Sevink G.J.A., "Model for pattern formation in polymer surfactant nanodroplets", Macromolecules 36 (21), 2003, pp. 7891-7983.

18. Fraaije J.G.E.M., vanVlimmeren B.A.C, Maurits N.M., et al, "The dynamic mean-field density functional method and its application to the mesoscopic dynamics of quenched block copolymer melts", J Chem Phys 106 (10), 1997, pp. 4260-4269.

19. Lyakhova K.S., Zvelindovsky A.V., Sevink G.J.A., et al, "Phase behavior in thin films of cylinder-forming ABA block copolymers: Mesoscale modeling", J Chem Phys. 120, 2004, pp. 1117-1126.

20. Li Y.M., Xu G.Y., Chen A.M., et al, "Aggregation between $\begin{array}{lllr}\text { Xanthan and } & \text { Nonyphenyloxypropyl } & \beta- \\ \text { Hydroxyltrimethylammonium } & \text { Bromide in Aqueous Solution: }\end{array}$ MesoDyn Simulation and Binding Isotherm Measurement”, J Phys Chem B. 109 (47), 2005, pp. 22290-22295.
21 Bai G., Nichifor M., Lopes A., et al, "Thermodynamic Characterization of the Interaction Behavior of a Hydrophobically Modified Polyelectrolyte and Oppositely Charged Surfactants in Aqueous Solution: Effect of Surfactant Alkyl Chain Length", J Phys Chem B 109 (1), 2005, pp. 518-525

22 Li Y.Y., Hou T.J., Guo S.L., et al, "The MesoDyn simulation of pluronic water mixtures using the "equivalent chain" method", Phys Chem Chem Phys, 2 (12), 2000, pp. 2749-2753.

23 Chen H.Y., Wu Y., Tan Y.Z., et al, "Mesoscopic simulation of surfactant/silicate self-assembly in the mesophase formation of SBA-15 under charge matching interactions", Eur Polym J 48, 2012, pp. 1892-1900.

24 Zheng L.S., Yang Y.Q., Guo X.D., et al, "Mesoscopic simulations on the aggregation behavior of $\mathrm{pH}$-responsive polymeric micelles for drug delivery", J Colloid Interface Sci 363, 2011, pp. 114-121. 